\section{Mycobacterium gordonae in Patient with Facial Ulcers, Nosebleeds, and Positive T-SPOT.TB Test Result, China}

\author{
Yanqing Chen, Juan Jiang, Haiqin Jiang, \\ Jun Chen, Xiaopo Wang, Weida Liu, \\ Zhiming Chen, Ying Shi, Wenyue Zhang, \\ Hongsheng Wang
}

Author affiliations: Institute of Dermatology, Chinese Academy of Medical Sciences and Peking Union Medical College, Nanjing, China (Y. Chen, J. Jiang, H. Jiang, X. Wang, W. Liu, Z. Chen, Y. Shi, W. Zhang, H. Wang); Jiangsu Key Laboratory of Molecular Biology for Skin Diseases and STIs, Nanjing, (Y. Chen, J. Jiang, H. Jiang, X. Wang, W. Liu, Z. Chen, Y. Shi, W. Zhang, H. Wang); Jiangsu Provincial Cancer Hospital, Nanjing (J. Chen)

DOI: https://dx.doi.org/10.3201/eid2307.162033

Mycobacterium gordonae is often regarded as a weak pathogen that only occasionally causes overt disease. We report a case of $M$. gordonae infection in the facial skin, nasal mucosa, and paranasal sinus in an immunocompetent patient and review previous cases. The T-SPOT.TB test

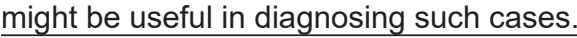

$\mathrm{N}$ ontuberculous mycobacteria are increasingly more involved in causing human infections. Mycobacterium gordonae, a type of slow-growing nontuberculous mycobacterium, is generally regarded as a weak pathogen, although it has caused some disease in humans (1-3). We describe a case of $M$. gordonae infection in the facial skin, nasal mucosa, and paranasal sinus of an immunocompetent patient in China.

In March 2016, a 60-year-old woman with no history of tuberculosis or immunosuppression sought treatment for a 2-year history of asymptomatic facial ulcers and intermittent nosebleeds. The primary lesion was a small erythema on the left cheek that, without incurring trauma, developed into an ulcer. The condition gradually worsened. One year before visiting our hospital, she received a diagnosis of lupus erythematosus; she had also been referred to otorhinolaryngology, where she was treated unsuccessfully with nasal endoscopic surgery. The skin lesions were erythematous, covered with yellow crusts, and on both sides of the patient's face (Figure, panel A). A nasal examination revealed small scabs in the nasal vestibule. Lymph nodes were not palpable.

Laboratory test results indicated the patient was negative for autoantibodies and HIV. A computed tomography (CT) scan of the paranasal sinuses showed ethmoid and maxillary sinusitis (Figure, panel B). Histologic examination indicated that a large number of lymphocytes, a few histiocytes, and plasma cells had infiltrated the lesion (Figure, panel E). Periodic acid-Schiff and acid-fast stains were negative for bacteria, and tissue culture was negative for fungus. Mycobacterial infection was suspected, but because mycobacteria are slow-growing, another test was needed to inform clinical practice. An alternative diagnostic test, the T-SPOT.TB (Oxford Immunotec Ltd., Oxford, England), an interferon- $\gamma$ release assay, was performed and showed positive results (tuberculosis $[\mathrm{TB}]$ antigen $-\mathrm{Nil}=20 ; \geq 6$ was positive). After 3 weeks of culture at $32^{\circ} \mathrm{C}$ in Löwenstein-Jensen medium, smooth, creamy, yolk-yellow bacteria were observed (Figure, panel F). Ziehl-Neelsen staining indicated they were acid-fast bacilli. Sequence analysis of $16 \mathrm{~S}$ rRNA revealed a $99 \%$ similarity with $M$. gordonae strain Y27, and the hsp65 gene showed a 100\% similarity with $M$. gordonae strain FJ09081. Further sequence analysis showed 16S rRNA was 99\% homologous and hsp65 $96 \%$ homologous with $M$. gordonae ATCC 14470. The diagnosis of $M$. gordonae infection was made.

After diagnosis, the patient was empirically treated with clarithromycin $(500 \mathrm{mg} / \mathrm{d})$ and moxifloxacin (400 $\mathrm{mg} / \mathrm{d})$. At the 4-month follow-up, no new nosebleeds or skin lesions were reported, and skin examination revealed scattered atrophic scars on the face (Figure, panel C). CT scans showed substantial improvement of the ethmoid and maxillary sinuses (Figure, panel D); T-SPOT.TB results were negative $(\mathrm{TB}$ antigen $-\mathrm{Nil}=0)$. Treatment continued for 2 more months.

A drug sensitivity test showed that the isolate from the patient was sensitive to clarithromycin, ethambutol, and moxifloxacin but less sensitive to rifampin, rifabutin, and isoniazid (4). No adverse drug reactions or recurrences were noted for up to 3 months after treatment was completed.

$M$. gordonae is found widely throughout the environment. Infections caused by $M$. gordonae usually occur in the lungs (and only occasionally in other organs) of immunocompromised patients. A cutaneous infection with $M$. gordonae is unusual and a paranasal sinus infection even rarer.

Seven cases of cutaneous infection with $M$. gordonae have been reported; all were in women 38-80 years of age. The infection can affect persons who have not experienced trauma or been exposed to immunosuppressants. The most common lesions caused by $M$. gordonae are nodules that slowly enlarge and become ulcerated over several months. Lesions usually are located on the face, at distal extremities, or at sites of previous trauma $(1,5-8)$. In this case, the infection caused lesions throughout the facial skin, nasal mucosa, and paranasal sinus, probably because the delay in accurate diagnosis allowed for wide dissemination of the pathogen.

Common laboratory methods for diagnosis of nontuberculous mycobacterial infection include histopathologic stainings, tissue culture, PCR, and gene sequencing. 

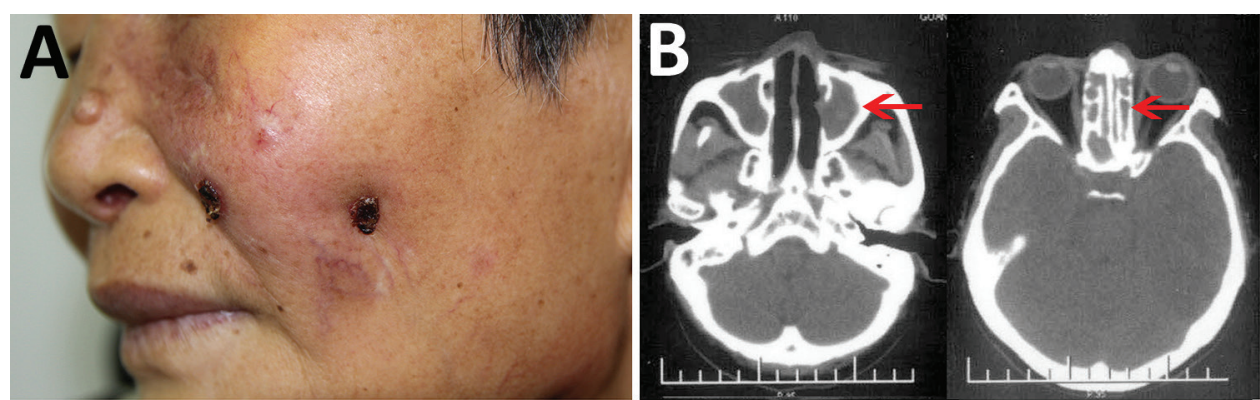

Figure. Mycobacterium gordonae infection in a 60-yearold immunocompetent woman, China. A) Facial lesions before treatment. Ulcers were erythematous and covered with yellow crusts. B) Computed tomography images before treatment show heterogeneous hypersignal in the ethmoid and left maxillary sinus (arrows).
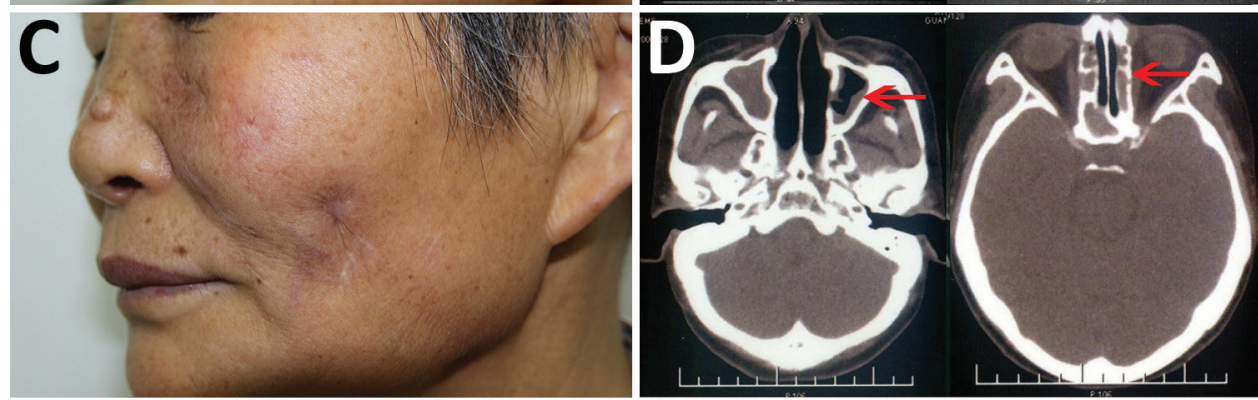

C) Facial lesions after treatment. Atrophic scars are seen at sites of previous lesions.

D) Computed tomography images after treatment show recovery of the ethmoid sinus and left maxillary sinus (arrows). E) Hematoxylin and eosin stain of nasal mucosa showing the infiltration of a large number of lymphocytes, a few histiocytes, and plasma cells. Scale bar

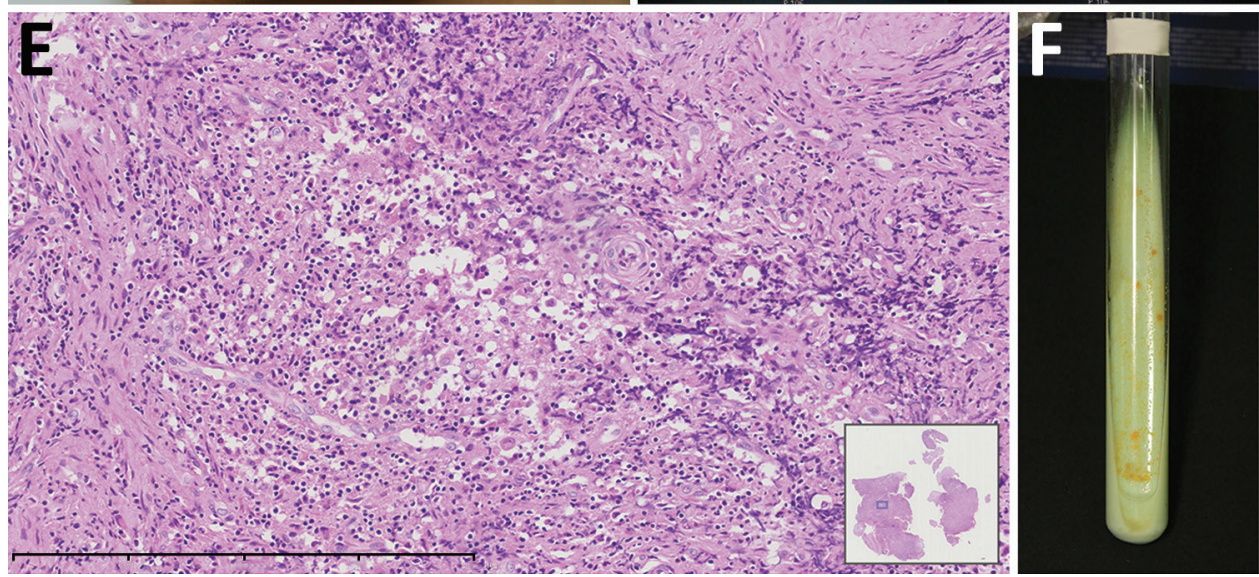
corresponds to $400 \mu \mathrm{m}$. Inset shows the nasal mucosa sample (original magnification $\times 20$ ). F) Tissue culture 3 weeks after incubation shows yolk-yellow bacteria growing in LöwensteinJensen medium.

Tissue culture and sequencing usually provide the most reliable evidence for diagnosis; however, tissue culture has a low sensitivity and is time-consuming, making early diagnosis difficult.

In this case, the initial patient sample was positive by T-SPOT.TB, and after mycobacterium-specific treatment and clinical improvement, the convalescent-phase patient sample was negative by T-SPOT.TB. These results were highly suggestive that the patient had a mycobacterial infection and that the infection was adequately treated.

Although it is generally believed that a positive TSPOT.TB result means the patient has an $M$. tuberculosis infection, positive results have been reported for infections caused by nontuberculous mycobacteria $(2,9)$. The T-SPOT.TB assay uses the $M$. tuberculosis antigens ESAT- 6 and CFP-10. The ESAT- 6 and CFP-10 genes are located within the $M$. tuberculosis region of difference 1 (RD1), a DNA sequence that is also present in M. marinum, M. kansasii, M. szulgai, and M. gordonae (10).
Because these genes are present in other mycobacterial genomes, the T-SPOT.TB assay might be useful for diagnosing infections with multiple RD1-possessing mycobacteria. However, further studies are needed to confirm its diagnostic value.

This manuscript was supported by grants from Natural Science Foundation of China (81371751) and Jiangsu Provincial Natural Science Foundation (BK20141065).

Dr. YanqingChen is a fellow of the Institute of Dermatology at the Chinese Academy of Medical Sciences and Peking Union Medical College. Her major research interests are nontuberculous mycobacterial infection and skin diseases.

\section{References}

1. Foti C, Sforza V, Rizzo C, De Pascale G, Bonamonte D, Conserva A, et al. Cutaneous manifestations of Mycobacterium gordonae infection described for the first time in Italy: a case report. Cases J. 2009;2:6828. http://dx.doi.org/10.4076/1757-1626-2-6828 
2. Gajurel K, Subramanian AK. False-positive QuantiFERON TB-Gold test due to Mycobacterium gordonae. Diagn Microbiol Infect Dis. 2016;84:315-7. http://dx.doi.org/10.1016/ j.diagmicrobio.2015.10.020

3. Asija A, Prasad A, Eskridge E. Disseminated Mycobacterium gordonae infection in an immunocompetent host. Am J Ther. 2011;18:e75-7. http://dx.doi.org/10.1097/MJT.0b013e3181e32e55

4. Clinical and Laboratory Standards Institute. Susceptibility testing of mycobacteria, nocardiae, and other aerobic actinomycetes; approved standard (M24-A). Wayne (PA): The Institute; 2003.

5. Gengoux P, Portaels F, Lachapelle JM, Minnikin DE, Tennstedt D, Tamigneau P. Skin granulomas due to Mycobacterium gordonae. Int J Dermatol. 1987;26:181-4. http://dx.doi.org/ 10.1111/ j.1365-4362.1987.tb00888.x

6. Rusconi S, Gori A, Vago L, Marchetti G, Franzetti F. Cutaneous infection caused by Mycobacterium gordonae in a human immunodeficiency virus-infected patient receiving antimycobacterial treatment. Clin Infect Dis. 1997;25:1490-1. http://dx.doi.org/ 10.1086/517006

7. Shelley WB, Folkens AT. Mycobacterium gordonae infection of the hand. Arch Dermatol. 1984;120:1064-5. http://dx.doi.org/10.1001/ archderm.1984.01650440094028

8. Zala L, Hunziker T, Braathen LR. Chronic cutaneous infection caused by Mycobacterium gordonae. Dermatology. 1993; 187:301-2. http://dx.doi.org/10.1159/000247275

9. Kuznetcova TI, Sauty A, Herbort CP. Uveitis with occult choroiditis due to Mycobacterium kansasii: limitations of interferon-gamma release assay (IGRA) tests (case report and mini-review on ocular non-tuberculous mycobacteria and IGRA cross-reactivity). Int Ophthalmol. 2012;32:499-506. http://dx.doi.org/10.1007/s10792-012-9588-3

10. Vordermeier HM, Brown J, Cockle PJ, Franken WP, Arend SM, Ottenhoff THM, et al. Assessment of cross-reactivity between Mycobacterium bovis and M. kansasii ESAT-6 and CFP-10 at the T-cell epitope level. Clin Vaccine Immunol. 2007;14:1203-9. http://dx.doi.org/10.1128/CVI.00116-07

Address for correspondence: Hongsheng Wang, Institute of Dermatology, Chinese Academy of Medical Sciences and Peking Union Medical College, St. 12 Jiangwangmiao, Nanjing, Jiangsu, 210042, China; email: whs33@vip.sina.com

\section{Norovirus GII.17 as Major Epidemic Strain in Italy, Winter 2015-16}

\author{
Giovanni Maurizio Giammanco, Simona De Grazia, \\ Floriana Bonura, Vincenzo Cappa, Sara Li Muli, \\ Arcangelo Pepe, Maria Cristina Medici, \\ Fabio Tummolo, Adriana Calderaro, \\ Francesca Di Bernardo, Piera Dones, \\ Anna Morea, Daniela Loconsole, \\ Cristiana Catella, Valentina Terio, \\ Krisztiàn Bànyai, Maria Chironna, Vito Martella
}

Author affiliations: Università di Palermo, Palermo, Italy (G.M. Giammanco, S. De Grazia, F. Bonura, V. Cappa, S. Li Muli, A. Pepe); Università degli Studi di Parma, Parma, Italy (M.C. Medici, F. Tummolo, A. Calderaro); ARNAS Ospedale Civico e Di Cristina, Palermo (F. Di Bernardo, P. Dones); Università Aldo Moro di Bari, Bari, Italy (A. Morea, D. Loconsole, M. Chironna); Università Aldo Moro di Bari, Valenzano, Italy (C. Catella, V. Terio, V. Martella); Hungarian Academy of Sciences, Budapest, Hungary (K. Bànyai)

DOI: http://dx.doi.org/10.3201/eid2307.161255

In winter 2015-16, norovirus Gll.17 Kawasaki 2014 emerged as a cause of sporadic gastroenteritis in children in Italy. Median patient age was higher for those with GII.17 than Gll.4 infection (55 vs. 24 months), suggesting limited cross-protection for older children.

Toroviruses are a major cause of acute gastroenteritis in children and adults. Although $>30$ norovirus genotypes can infect humans, the genotype GII.4 has been associated with most norovirus-related outbreaks and sporadic cases of gastroenteritis since the mid-1990s. Norovirus strains undergo genetic and antigenic evolution through accumulations of point mutations and intragenotype and intergenotype recombination.

During the winter season 2014-15, a novel NoV GII. P17_GII.17 variant, Kawasaki 2014, emerged in several countries in Asia, replacing the previously dominant variant, GII.4 Sydney 2012 (1-4). Kawasaki 2014 is phylogenetically distinct (Kawasaki-308 lineage) from earlier GII.17 variants that circulated in 2013 and 2014 and from the original 1978 GII.17 strain, which had a GII.4 open reading frame (ORF) 1 gene (4). The spread of the variant Kawasaki 2014 in Asia was unexpected because this was the first time a variant other than GII.4 acquired such epidemiologic relevance. Until now, the Kawasaki 2014 variant had been reported in a limited number of cases in countries outside of Asia $(5,6)$, including some in Europe (6-8), with most cases occurring sporadically.

The Italian Study Group for Enteric Viruses (http://isgev.net) monitors the epidemiology of enteric viruses in children through hospital-based surveillance in 3 geographically distinct areas: northern Italy, in Parma (University Hospital); southern Italy, in Bari (Pediatric University Hospital "Giovanni XXIII"); and on Sicily island, in Palermo (ARNAS Civic Hospital). The official total pediatric population ( $0-14$ years of age) includes 426,569 newborns, infants, and children (as of January 1, 2015).

From January 2015 through February 2016, the Italian Study Group for Enteric Viruses analyzed 2,603 fecal samples of children ( $0-14$ years of age) with diarrhea. 